\title{
Key technology of research anti-interference simulation system of parameters complex electromagnetic environment
}

\author{
GAO Ying ${ }^{1}$, WANG Amin ${ }^{1}$, ZHANG Jinhui ${ }^{1}$, GUO Shuxia ${ }^{2}$ \\ ${ }^{1}$ Northwestern Polytechnic University, School of Marine Engineering, Xi'an 710072, P. R. China \\ ${ }^{2}$ Northwestern Polytechnic University, Science and Technology on UAV Laboratory, Xi'an710065, P. R. China \\ 'gaoying@nwpu.edu.cn2270890382@qq.com
}

\begin{abstract}
This paper is about anti-interference semi-physical simulation system design issues based on the integrated darkroom and instrument, this simulation system gives us an anti-interference simulation system of parameters complex electromagnetic environment based on parametric design and rapid construction, researches quickly build and mapping method for simulation scene and instrument based on a script control and other key technologies the simulation platform can achieves complex electromagnetic environment signal transmitter and performance verification communication ability of electromagnetic environment simulation of UAV data link through the parameterized design and control of the interference signal. The simulation system fast constructs the indoor wireless test environment using the script editor and graphical interface, the scene has a dynamic editing, a function of quickly build and recording test data, so easy to test analysis and evaluate the test results of the device in the form of a map. Parameters of complex electromagnetic anti-interference system has complex electromagnetic environment simulation capabilities of multiplexer interference source system with multi-channel and a variety of signals, a good user interface and scalability through the control of application and management software and instrument. Complex electromagnetic environment parameters of anti-interference simulation results show that parametric design and control method is effective to improve the analog performance of UAV data link and testing communication proficiency. It has better theoretical research and application value.
\end{abstract}

Index Terms- UAV, data link, anti-interference, semi-physical simulation

\section{Introduction}

Electronic warfare intensifying in modern warfare, the battlefield electromagnetic space environment has become the key factor restricting the development trends of the aviation communication system increasingly. There is more and more meticulous and exhaustive research in the simulation work of various the battlefield electromagnetic space environment simulations.

The Reference[1] mentioned modern UAV in the war of defense measures, it proposes UAV facing the complex electromagnetic environment is more important, the Reference[2] provided the relevant points of the semi-physical simulation system structures, and pointed out goodness of the physical simulation; Over the previous work it can be seen UAV facing the electromagnetic environment simulation. Here we present the complex electromagnetic environment which is based on parametric design of anti-interference simulation system platform combining the advantage of semiphysical simulation.

The system is anti-interference semi-physical simulation system from the point of view of the simulation, which established a simulation platform for electronic warfare, a realistic reproduce and simulate electronic warfare has taken a pioneering step from the point of view of the system platform. The platform completes the following functions: complex electromagnetic environment signal transmitter; the performance verification of data chain simulation; expression the impact of the electromagnetic environment UAV communication capabilities in the form of a map; then assessment, inspection and analysis the UAV a variety of links and battlefield communication environment, therefore it has a high economic efficiency ${ }^{[3]}$. The system integrities research indoor wireless the anti-interference test environment system integration, system integration principle reasonable, effectively splits the system functions system into relatively independent functional modules, the interface between modules is clear, in order to integrate system and functional extensions conveniently, it is using a combination of proven technology with new technology, and to ensure system integration the effectively, novelty, flexibility and scalability.

We research anti-interference test methods and test platform receiving for receiving terminal indoor to ensure that the testing equipment anti-interference requirements of the receiving, as well as test environment system features, technical specifications to meet the requirements, using the script editor and graphical interface, fast simulation indoor wireless test environment, the scenes with dynamic editing, and rapid construction of feature, recording and reproduction the test data in order to analysis and evaluate the testing results of the test device under easily.

\section{System Introductions}

Complex electromagnetic environment simulation system is controlled by the master control management software, the entire system of hardware and software connected each other with the communication network system [4]. The overall framework of the system is based on total control management software as the core, the system functions are divided into the complex electro- magnetic environment simulation control, 
data interface, interference source management and interference simulation database, system assessment and other functional modules.

The system components are as follow:

1) Total control management software

Total control management software completes the construction of the test scenarios, parameter setting, test preparation, test process control, test data processing functions.

2) Electromagnetic environment simulation control

Complicated electromagnetic environment simulation complete simulation and control of multiple, different forms of electromagnetic signals.

3) Data Interface

The test scene data from total control management software form controlled information file or database which is available for complex electromagnetic environment simulation.

4) Interference source management

Management expanded the types of interference analog equipment in the system, included the establishment, change attributes, delete, etc.

5) Interference simulation database

Interference simulation database includes two aspects: 1 is a form of expect interference 2 the forms of interference can be simulated by existing meter, and both of all will be managed by database ,complex electromagnetic environment simulation purpose is the objective existence electromagnetic signal be simulated by an analog device.

Fig. 1 is the block diagram of the complex environment simulation and control software system.

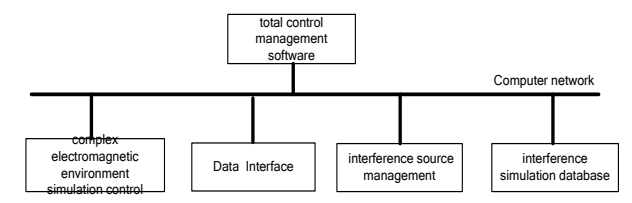

Fig.1 Environment simulation and control software system block diagram

\section{A. System Hardware}

Complicated electromagnetic environment simulation system hardware consists of a computer control system, interfere source device, optical fiber communication module, multiple input multiple output microwave switches, microwave, cable and accessories, and its hardware configuration is shown in Fig.2:

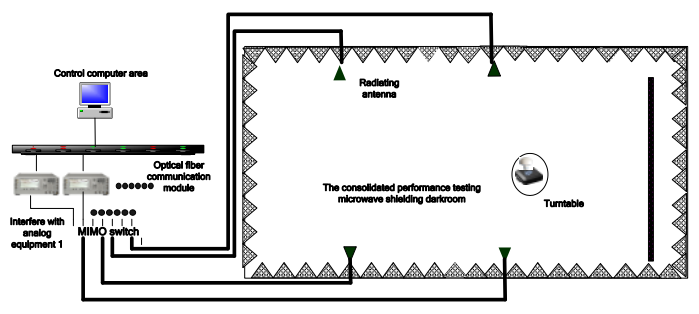

Fig.2 Hardware working Layout
The system hardware components are as follow:

1) The computer control system

The computer control system is used to control the normal operation of the whole system, completes manage- ment control of the systems, data storage and other functions. Including the host computer, device control computer;

2) Interfere source device

Interfere source device to be controlled by the master control computer system properly simulates all kinds of interference simulation instruction issued by the master control computer task, provided by the R \& S system.

3) Fiber-optic communication module

Fiber-optical communication system establishes the network connection between the each device computer and analog instrumentation; realizes the exchange of data between devices, and ensures the smooth flow of the entire simulation test system data.

4) Multiple input multiple output microwave switch

In order to realize complicated electromagnetic environment simulation system in an anechoic chamber, we need to simulate interference sources based on user testing requirements radiation from any radiation port, intended to configure a programmable multi-input multiple-output microwave switch.

5) Microwave cables and accessories

High performance RF cable and adapter, to make sure RF link system is working properly.

\section{B. Software Control Flow}

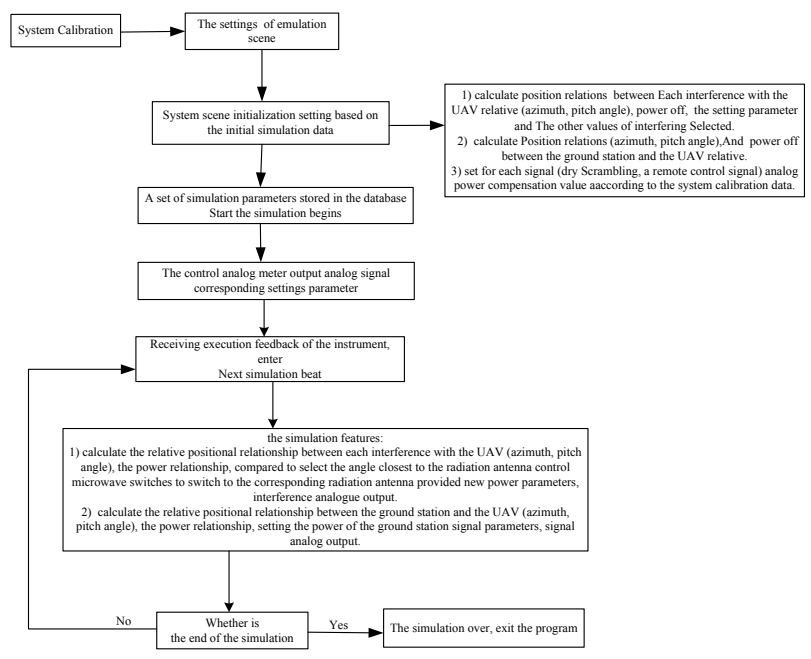

Fig.3 Complex electromagnetic environment simulation flowcharts

System simulation begins with system initialization, system calibration process includes UAV flight angle in the whole system simulation process, the interference, checks and calibrate the signal power and the network connection status, simulation scenarios setting adds a variety of simulation elements dynamically in terrain scene of simulated battlefield, including UAV, the interference source, ground stations, etc; large terrain will be selected Transform according to the needs [5], and then set the relevant parameters of the interference and communication signals, complete the set of initialization 
values, the initial value of these parameters are imported into the database of interference and the instrument types, then we calculate angle and pitch angle coordinate relations solutions of interference sources, ground station and UAV ${ }^{[6]}$. Setting the calculated the signal power value of UAV receiving terminal according to the initial frequency value of the ground station and interference; setting compensation power value of the every analog signal (interference, remote control signal). Entire complex electromagnetic environment simulation flowchart is as shown in Fig.3.

\section{Key Technologies}

This software is based on the pivotal semi-physical simulation, the instrument control is pivotal in software. In the process of the system work, we need to know the instrumentation connected to the current host computer in accordance with the demand of the practical application. In the initial produce, users can intuitively watch the currently available instrumentation, the instrumentation required to change and improvement before the engineering test.

When software is running, click the instrument scan button in the following dialog box, the system will start to scan hardware devices, based on the above analysis, we need to first scan the instrumentation connected to the host computer, because the device number connected with the host computer will be different depending on the experimental, if the number of devices connected to the host computer's too much, it will increase the time of the scanning process, which will increase the user's waiting time. So we increase the progress bar to display the current progress of the scan, the scan results show the following list box shown in Fig.4:

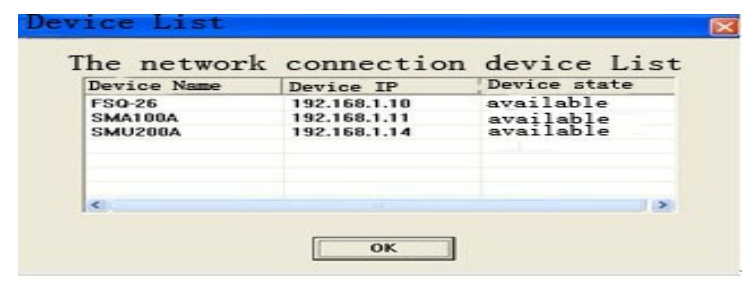

Fig.4 list box-lists the scan to the device

The system traverse the address of 256 IP (192.168.1.0 192.168.1.255), after traverse 256 IP address filter operation, screen instrumentation equipment in 256 IP addresses to connect to the host computer, while the other is not connected to the host computer's IP address shield.

Test initialization is complete, the scan list box displays the resulting instrument name (such as SMU200A), IP address, and the available state information, this information will be stored in the previously established database, interference setting set in the interfering dialogue box, the system will save the parameters of various types of interference into the database at the same time issue the setting parameter to the instrumentation equipment, and then produce a particular interference signal by the instrument, these interference signals eventually are send out by the antenna, analog generate interference signal.
The present system is corresponding to the different instruments for the different types of interference, and the Intelligent software displays the available instrumentation, then by the compare the same name of the instrument name and script name to load the corresponding instruments matching the script file ${ }^{[7]}$, while, the script file contains a large number of the a large number of instrument parameters and driver instrumentation when driving instruments. The logic flow diagram is shown in the Fig.5:

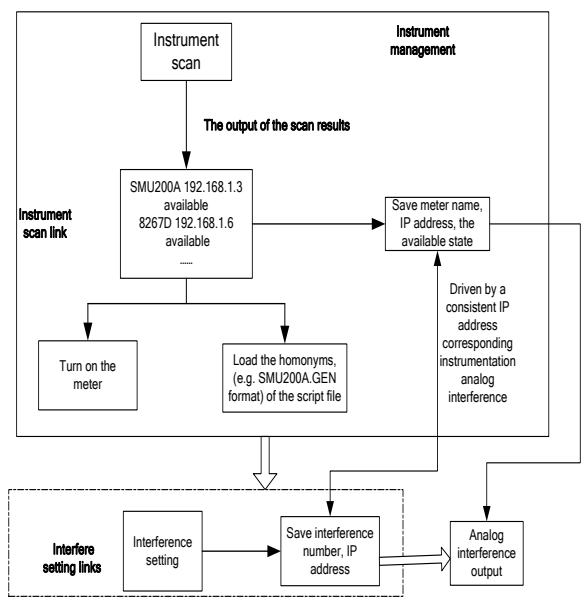

Fig. 5 meter management processes

Here we present system has defined a variety of types of instruments corresponding to interference in the database, which is as shown Fig.6:

\begin{tabular}{|c|c|c|c|c|c|c|c|}
\hline connuy send & conniy yeur & mainei & manise & nuise & pulse & SWEP & waye \\
\hline 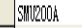 & Io & SMMODOh & SMll200h & SIll1200h & Inlle2010h & Smlle200h & SMllo:OOA \\
\hline 882670 & no & B8267D & Io & B8285T & I0 & B82651 & B8267D \\
\hline no & $\mathrm{PSO}-26$ & Io & In & Ii & Io & In & no \\
\hline SWH100h & Ino & SUNP:00h & SMl1:100h & SIP $100 h$ & Sill100k & SWrr100h & SMIP100h \\
\hline Ind100h & Io & Slllilook & SMl100h & Sllli100h & SIlld100\% & Sllli100h & SIMH $100 \mathrm{O}$ A \\
\hline * & & & & & & & \\
\hline
\end{tabular}

Fig. 6 the microwave instrument defined in a database

In the interference settings dialog box, we select the dropdown list box of the initialize instrument according to the figure data table above, in the same trial, if an instrument has been used, the corresponding instrument in the program is marked "has been used" in the subsequent interference initialization, we select the instrument according to the data table to initialize the drop-down list box, check each instrument, if the instrument marked "has been used", the meter delete, then there are no the instrument in the instrument class table box, realize the intelligent management of the instrument.

For different interference and signal, respectively, driven by a different type of instrument, system select the instrument and call the appropriate instrument script ${ }^{[8]}$, loading the corresponding instrument driver script, further to save processing time and improve the system corresponding speed, to enhance the efficiency of the analog microwave instrument, and to complete the effective management of instruments. 
In the system, the dynamically complete instrument type to be added, and the selection of the instrument ID. Different instrument and interference require for the different types of script files and drivers ${ }^{[9]}$. There are two ways to add the interference signal source; one of them can be namely the traditional way, the other named scrip driver.

Adding the process of the interference signal source in a conventional manner, it must define different remote command according to different type of signal source in the remote control, so here the program control design must be specific signal source, as shown in Fig.7. Therefore, the conventional way to add the interference signal source will increase the complexity of the system, and increase the limitations for the expansion of the system, et. .

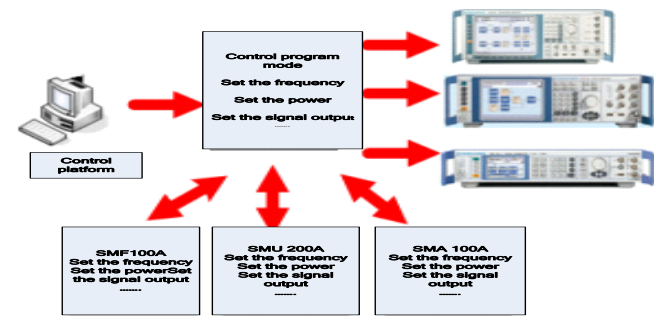

Fig. 7 the script flexible way to add the interference source

Due to the shortcomings of the conventional manner to add the interference signal source. In the system, we add script file of source control, so simplify the process when adding different types of sources provided by different manufacturers, thereby reducing the repeatability of the driving instruction, and improve the efficiency of the implementation of the drive instrumentation.

The frequency range of the source, other key interference parameter and control signal source command specified in the script file, it is possible to call the relevant function in the program directly and generate the target analog interference source signal to drive instrument ${ }^{[10]}$. The definition of the parameters and the instruction calls of the script files shown in Fig.8:

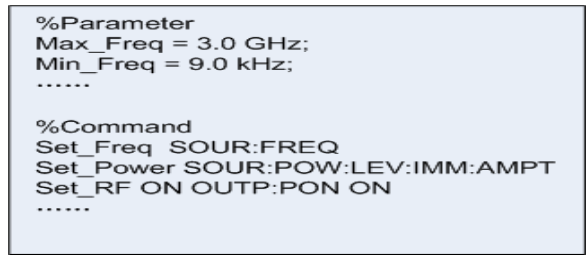

Fig. 8 The script files of the instruction

Now further we detailed descript the entire process of using a script to driver instrumentation, for example instrument SMU200A, here the main drive to the center frequency, the form of the signal, code rate, filter type, and filter coefficients. The first, write instrument SMU200A script file, the script file is a txt file. the written format: Set Carry Freq \$: FREQ\%f KHz@, then define structure variable script information, used to store and read the relevant parameters of the script file; the second, write script driver function
OnCommuPro, assigned the corresponding parameter value to the instrument in this function.

\section{Summaries}

The paper details the significance and the value of the parametric complex electromagnetic anti-interference system from the view of the overall system, the key technology of software and hardware components and project design, the system design should try to meet design requirements of signal fidelity and interference-fidelity. signal fidelity and interference comprehensiveness, should be involved each kinds of possible sources of interference in UAV as well as typical application scenarios; flexibility and the overall project program design principles flexibility easily control the operating mode of the system and operating parameters; scalability. With the escalating electronic warfare systems, complex electromagnetic environment interference signal and the test scenarios in the project simulation should be designed scalability, fidelity mainly considers simulation emission source in the airspace distribution of airspace changes, the dynamic range of indicators agreement with the actual situation.

\section{Acknowledgment}

The authors would like to thank anonymous reviewers for their helpful comments and suggestions on this paper.

\section{References}

[1] Zhang DM, Li Ep, David Yeo, Chow WS and James Quek, "Influences of Loading Absorber on the Performances of a Reverberation Chamber," International Symposium on Electromagnetic Compatibility, vol. 1, pp. 279-281, August 2003.

[2] Rajamani V., Bunting CF and West, JC, "Calibration of a Numerically Modeled Reverberation Chamber," International Symposium on Electromagnetic Compatibility, pp. 87-91, August 2009.

[3] Arnaut, Luk., "Operation of Electromagnetic Reverberation Chambers with Wave Diffractors At Relatively Low Frequencies," IEEE Transactions on Electromagnetic Compatibility, vol. 43,no. 4, pp. 637 653, November 2001.

[4] Arnaut, Luk., "Effect of Size, Orientation and Eccentricity of Mode Stirrers On Their Performance in Reverberation Chambers," IEEE Transactions on Electromagnetic Compatibility, vol. 48, no. 3, pp. 600602, August 2006.

[5] Zhao chou at SIFA, Zhao Ying-kun tactical communications training electromagnetic environment simulation and simulation research [J] Journal of Hebei University of Science and Technology, vol. 32, no. 8, pp.175-177, 2011.

[6] Yang Fang-fang, Yin Ya-lan, Sheng Qian-sheng. Modeling and simulation on the network of TADIL vol. 10, no. 2, pp. 28-31,2012.

[7] Qin Da-guo, Li-bo ,Chen Xiao-wu, Zhao Hong-li .Design and Implem entation Platfo rm for Satellite of Semi-physical SimulationCom munication Links . vol. 6, no. 27, pp. 49-52,2012.

[8] Yin Ya-lan, Yang Fang-fang, Sheng Qian-sheng. Modeling and simulation on the network of TADIL vol. 4, no. 2, pp. 67-70.2010.

[9] Wang Hong-yang. Research on the Electromagnetic Environment Faced by Surface to Air M issile Operations and the Countermeasures. vol. 16, no. 3, pp. 37-41, 2012.

[10] Paul Hallbjorner, Ulf Carlberg, Kent Madsen and Johan Andersson, "Extracing Electrical Material Parameters of Electrically Large Dielectri Objects from Reverberation Chamber Measurements of Absoption Cross Section," IEEE Transactions on Electromagnetic Compatibility, vol. 47, no. 2, pp. 291-303, May 2005. 